\title{
Large-Eddy Simulation of Stably-Stratified Flow Over a Steep Hill
}

\author{
Feng Wan · Fernando Porté-Agel
}

Received: 26 March 2010 / Accepted: 29 October 2010 / Published online: 13 November 2010

(C) Springer Science+Business Media B.V. 2010

\begin{abstract}
Large-eddy simulation (LES) is used to simulate stably-stratified turbulent boundary-layer flow over a steep two-dimensional hill. To parametrise the subgrid-scale (SGS) fluxes of heat and momentum, three different types of SGS models are tested: (a) the Smagorinsky model, (b) the Lagrangian dynamic model, and (c) the scale-dependent Lagrangian dynamic model (Stoll and Porté-Agel, Water Resour Res 2006, doi:10.1029/ 2005WR003989). Simulation results obtained with the different models are compared with data from wind-tunnel experiments conducted at the Environmental Flow Research Laboratory (EnFlo), University of Surrey, U.K. (Ross et al., Boundary-Layer Meteorol 113:427-459, 2004). It is found that, in this stably-stratified boundary-layer flow simulation, the scaledependent Lagrangian dynamic model is able to account for the scale dependence of the eddy-viscosity and eddy-diffusivity model coefficients associated with flow anisotropy in flow regions with large mean shear and/or strong flow stratification. As a result, simulations using this tuning-free model lead to turbulence statistics that are more realistic than those obtained with the other two models.
\end{abstract}

Keywords Large-eddy simulation - Stable stratification · Subgrid-scale modelling · Two-dimensional hill

\section{Introduction}

Large-eddy simulation (LES) has become a popular tool to study turbulent atmospheric boundary-layer (ABL) flows over topography. In particular, several LES studies have

F. Wan · F. Porté-Agel ( $\varangle)$

Saint Anthony Falls Laboratory, Department of Civil Engineering, University of Minnesota,

2 Third Avenue SE, Minneapolis, MN 55414, USA

e-mail: fernando.porte-agel@epfl.ch

F. Porté-Agel

School of Architecture, Civil and Environmental Engineering, École Polytechnique Fédérale de Lausanne, 1015 Lausanne, Switzerland 
investigated the effect of topography on boundary-layer turbulence under neutral stratification conditions (e.g., Gong et al. 1996; Henn and Sykes 1999; Brown et al. 2001; Calhoun and Street 2001; Iizuka and Kondo 2004; Wan et al. 2007). To this date, however, only a few studies have used LES to study stably-stratified boundary-layer flows over topography (e.g., Calhoun et al. 2001), and not much is known about the accuracy of the LES technique and subgrid-scale (SGS) models in simulations of this type of flow. Considering the sensitivity of LES results to SGS parametrisations in simulations of the neutral boundary layer over topography (e.g., Iizuka and Kondo 2004; Wan et al. 2007) and the stable boundary layer over flat terrain (e.g., Beare et al. 2006; Stoll and Porté-Agel 2008), one expects the combined effects of topography and atmospheric stability to pose an important challenge to SGS models in the LES.

LES resolves scales of turbulent transport larger than the grid/filter scale $\Delta$, and parametrises smaller (subgrid) scales using a SGS model. The most commonly used SGS models in the large-eddy simulation of ABL flows are the eddy-viscosity model for the SGS stresses and the eddy-diffusivity model for the SGS scalar fluxes. The specification of SGS model coefficients is one of the biggest challenges for LES as it has been found to have an important effect on the performance of the SGS models and, as a result, on the accuracy of the simulations. SGS coefficients are well established for homogeneous, isotropic turbulence (e.g., Lilly 1967; Mason and Derbyshire 1990). However, in flow regions with strong anisotropy at the smallest resolved and subgrid scales, the coefficients in the eddy-viscosity and eddy-diffusivity models are found to deviate from the well-established isotropic values (e.g., Deardorff 1970; Moin and Kim 1982; Bardina et al. 1983; Piomelli et al. 1988; Horiuti 1993; Canuto and Cheng 1997; Porté-Agel et al. 2001a; Kleissl et al. 2003; Porté-Agel 2004; Stoll and Porté-Agel 2006). In particular, the optimal values of those coefficients have to decrease in order to account for the local reduction in the characteristic length scales of the turbulence associated with increased mean shear near the surface (e.g., Kleissl et al. 2003; Stoll and Porté-Agel 2006) and/or increased atmospheric stability (e.g., Porté-Agel et al. 2001a; Kleissl et al. 2003).

The dynamic procedure provides a systematic approach to specifying the values of the SGS coefficients in the eddy-viscosity and eddy-diffusivity models (Germano et al. 1991; Moin et al. 1991; Lilly 1992). It does not require parameter specification or tuning, and model coefficients are optimised at every position and timestep based on information from the resolved scales, with the assumption that the coefficients are scale invariant in a range of scales between the filter/grid scale and a slightly larger test filter scale. In simulations of the neutral ABL flow over homogeneous surfaces, Porté-Agel et al. (2000) found that the dynamically calculated model coefficients showed a dependence on resolution (filter scale), which is against the underlying assumption of scale invariance made in the dynamic model. With this unreasonable assumption, the standard dynamic model has been shown to yield model coefficients that are too low. This, in turn, leads to an accumulation of energy at high wavenumbers and explains why the dynamic model is not dissipative enough and yields inaccurate turbulence statistics in simulations of ABL flow over homogeneous (PortéAgel et al. 2000) and heterogeneous (Bou-Zeid et al. 2005; Stoll and Porté-Agel 2006) flat surfaces, as well as neutral boundary-layer flow over topography (Iizuka and Kondo 2004; Wan et al. 2007). Motivated by those results, Porté-Agel et al. (2000) and Porté-Agel (2004) introduced the scale-dependent dynamic model, which is used to compute dynamically not only the value of the model coefficients in the eddy-viscosity and eddy-diffusivity models, respectively, but also the dependence of these coefficients with scale. To achieve that, the scale-dependent dynamic model assumes a power-law dependence of the coefficient with scale. It is important to note that this power-law dependence is only applied to a small range 
of resolved scales (between the filter scale and a test filter scale) and has been shown to be a realistic assumption in a priori field studies (Porté-Agel et al. 2001b; Bou-Zeid et al. 2008). Simulations of neutral boundary-layer flow over both flat surfaces (Porté-Agel et al. 2000; Porté-Agel 2004; Bou-Zeid et al. 2005; Stoll and Porté-Agel 2006) and simple topography (Wan et al. 2007) have shown that the scale-dependent dynamic model improves the level of SGS dissipation (rate of energy transfer from the resolved to the subgrid scales), which results in more realistic simulated velocity and scalar statistics.

In order to ensure numerical stability, the application of the dynamic and scale-dependent dynamic models requires some sort of averaging. For flows with directions of homogeneity, the averaging can be done over those directions (e.g., over horizontal planes in the case of flow over a flat homogeneous surface). For cases of flow over complex terrain, Lagrangian averaging (over flow pathlines) has been used in the Lagrangian dynamic model (Meneveau et al. 1996) as well as the scale-dependent Lagrangian dynamic model (Stoll and Porté-Agel 2006).

In this paper, we test the performance of three SGS models in simulations of stable boundary-layer flow over a single steep hill against laboratory experiments. These models are: (a) the Smagorinsky model, (b) the Lagrangian dynamic model, and (c) the recently developed scale-dependent Lagrangian dynamic model (Stoll and Porté-Agel 2006). Next, a brief description of the three models is given.

\subsection{The Smagorinsky Model}

The SGS stress $\tau_{i j}$ is commonly parametrised using an eddy-viscosity model of the form (Smagorinsky 1963):

$$
\begin{gathered}
\tau_{i j}-\frac{1}{3} \delta_{i j} \tau_{k k}=-2 \nu_{T} \widetilde{S}_{i j}, \\
v_{T}=\left[C_{S} \Delta\right]^{2}|\widetilde{S}|,
\end{gathered}
$$

where $\tau_{i j}$ is the SGS stress at filter (grid) scale, $\widetilde{S}_{i j}=\frac{1}{2}\left(\frac{\partial \widetilde{u}_{i}}{\partial x_{j}}+\frac{\partial \widetilde{u}_{j}}{\partial x_{i}}\right)$ is the resolved strainrate tensor, $v_{T}$ is the eddy viscosity, $|\widetilde{S}|=\left(2 \widetilde{S}_{i j} \widetilde{S}_{i j}\right)^{\frac{1}{2}}$ is the magnitude of the resolved strain-rate tensor, $C_{S}$ is the Smagorinsky coefficient, and $\Delta$ is the size of the spatial filter.

The value of the Smagorinsky coefficient $C_{S}$ is well established for isotropic and homogeneous turbulence ( $C_{S} \approx 0.17$, Lilly 1967 ). The reduction of the characteristic length scale and flow anisotropy near the surface, however, makes the optimal value of the Smagorinsky coefficient depart from its isotropic counterpart (e.g., Deardorff 1970; Moin and Kim 1982; Bardina et al. 1983; Piomelli et al. 1988). To account for this effect, Mason and Thomson (1992) proposed the following relation,

$$
\frac{1}{\lambda^{n}}=\frac{1}{\lambda_{o}^{n}}+\frac{1}{\left[\kappa\left(z+z_{o}\right)\right]^{n}},
$$

where $\kappa(\approx 0.4)$ is the von Karman constant, $\lambda=C_{S} \Delta$ is the length scale in the model, $\lambda_{o}=C_{o} \Delta$ is the length scale far from the wall, $z_{o}$ is the roughness length, and $C_{o}$ and $n$ are adjustable parameters. $C_{o}$ generally takes values between 0.1 and 0.3 , and $n$ is an integer often chosen to be 1,2 or 3 . In this study, the values of $C_{o}$ and $n$ are set to be 0.1 and 1, respectively. Recent field experiments (Porté-Agel et al. 2001a; Kleissl et al. 2003) and numerical simulations using dynamic models (Kleissl et al. 2006) have shown that the optimal value of the Smagorinsky coefficient decreases with increasing stability in order to account 
for the reduction of turbulence eddy sizes as well as characteristic length scales associated with thermal stratification. Even though some empirical formulations have been proposed to account for this effect (e.g., Kleissl et al. 2003), they are usually based on horizontallyaveraged variables and thus are not easily applicable to simulations of the heterogeneous boundary layer.

To parametrise the SGS scalar flux $q_{i}$, the eddy-diffusivity model is typically used:

$$
\begin{gathered}
q_{i}=-D_{T} \frac{\partial \tilde{\theta}}{\partial x_{i}}, \\
D_{T}=C_{S}^{2} \operatorname{Pr}_{\text {sgs }}^{-1} \Delta^{2}|\widetilde{S}|,
\end{gathered}
$$

where $q_{i}$ denotes SGS scalar flux at filter (grid) scale, $D_{T}$ is the eddy diffusivity, $C_{S}^{2} P r_{s g s}^{-1}$ is a lumped eddy-diffusivity coefficient composed of the Smagorinsky coefficient and the SGS Prandtl number $P r_{s g s}$. A value of 0.4 is often used for the SGS Prandtl number in neutral turbulent flow (e.g., Kang and Meneveau 2002) as well as the thermally stratified boundary layer (e.g., Kleissl et al. 2006). In our study, the value of the SGS Prandtl number is set to be 0.4 as well. However, it is important to note that recent experimental (e.g., Porté-Agel et al. 2001a; Kleissl et al. 2003) and numerical (e.g., Beare et al. 2006; Stoll and Porté-Agel 2008) studies have shown that $\operatorname{Pr}_{s g s}$ should increase with increasing atmospheric stability. This effect further contributes (together with the above-mentioned decrease in $C_{S}$ ) to a reduction in the lumped eddy-diffusivity coefficient and the model length scale in thermally stratified regions of the flow.

\subsection{The Lagrangian Dynamic Model}

The dynamic procedure (Germano et al. 1991) optimises the value of the Smagorinsky coefficient $\left(C_{S}^{2}\right)$ using information from the smallest resolved scales without the need for parameter tuning. The Germano identity is introduced as

$$
L_{i j} \equiv T_{i j}-\bar{\tau}_{i j}=\overline{\widetilde{u}_{i} \widetilde{u}_{j}}-\overline{\widetilde{u}}_{i} \overline{\widetilde{u}}_{j},
$$

where $L_{i j}$ is a resolved stress tensor, also referred to as the Leonard stress, and $T_{i j}$ is the SGS stress at a test filter scale $\bar{\Delta}$ (typically $\bar{\Delta}=2 \Delta$ ). The overbar in Eq. 6 denotes a spatial filtering operation at scale $\bar{\Delta}$. $T_{i j}$ can be calculated using the eddy-viscosity model

$$
T_{i j}-\frac{1}{3} \delta_{i j} T_{k k}=-2\left(C_{S}(\bar{\Delta}) \bar{\Delta}\right)^{2}|\widetilde{S}| \widetilde{S}_{i j},
$$

where $C_{S}(\bar{\Delta})$ denotes the Smagorinsky coefficient at the test filter scale $\bar{\Delta}$. Substituting the SGS stresses at scale $\bar{\Delta}$ (Eq. 7) and scale $\Delta$ (Eq. 1) into the Germano identity, one can calculate the error incurred by using the Smagorinsky model in the Germano identity as

$$
e_{i j}=L_{i j}-\frac{1}{3} \delta_{i j} L_{k k}-C_{S}^{2}(\Delta) M_{i j}
$$

where

$$
M_{i j}=2 \Delta^{2}\left(\overline{|\widetilde{S}| \widetilde{S}_{i j}}-4 \beta|| \widetilde{S} \mid \widetilde{S}_{i j}\right) .
$$

$\beta$ in Eq. 9 is the ratio of the model coefficients squared at the test filter scale and at the filter scale, i.e.,

$$
\beta=C_{S}^{2}(2 \Delta) / C_{S}^{2}(\Delta)
$$


The original (scale-invariant) dynamic model is based on the assumption that the coefficient is scale invariant and, consequently, $\beta=1$.

Using the least squares method to minimise the error given by Eq. 8, one can obtain the optimal value of $C_{S}^{2}$ as

$$
C_{S}^{2}=\frac{\left\langle L_{i j} M_{i j}\right\rangle}{\left\langle M_{i j} M_{i j}\right\rangle} .
$$

When implementing the dynamic model, one needs to perform some sort of averaging to ensure numerical stability. Here we use Lagrangian averaging (averaging backward over local fluid pathlines) as proposed by Meneveau et al. (1996). Only information at the previous and current timesteps is stored for model coefficient calculation. This Lagrangian averaging scheme is well suited for simulations of flow over complex terrain, where the flow is non-homogeneous.

For the SGS scalar flux, the Germano identity takes the following form

$$
K_{i}=Q_{i}-\bar{q}_{i}=\overline{\widetilde{u}_{i}} \widetilde{\tilde{\theta}}-\overline{\widetilde{u}}_{i} \overline{\widetilde{\theta}},
$$

where $Q_{i}$ is the SGS flux at the test-filter scale $\bar{\Delta}$. Using the eddy-diffusivity model, $Q_{i}$ can be calculated as

$$
Q_{i}=-\left[C_{S}^{2}(\bar{\Delta}) P r_{s g s}^{-1}\right] \bar{\Delta}^{2}|\overline{\widetilde{S}}| \frac{\partial \overline{\tilde{\theta}}}{\partial x_{i}} .
$$

Combining Eqs. 4, 12 and 13, one obtains the error incurred by using the eddy-diffusivity model in the Germano identity for scalar transport

$$
e_{i}=K_{i}-C_{S}^{2} \operatorname{Pr}_{s g s}^{-1}(\Delta) X_{i}
$$

where

$$
X_{i}=\Delta^{2}\left(\overline{|\widetilde{S}| \frac{\partial \tilde{\theta}}{\partial x_{i}}}-4 \beta_{\theta}|\widetilde{\widetilde{S}}| \frac{\partial \overline{\tilde{\theta}}}{\partial x_{i}}\right) .
$$

Here $\beta_{\theta}$ is a scale-dependence parameter defined as

$$
\beta_{\theta}=C_{S}^{2} \operatorname{Pr}_{\text {sgs }}^{-1}(2 \Delta) / C_{S}^{2} \operatorname{Pr}_{\text {sgs }}^{-1}(\Delta) .
$$

As in the case of $C_{S}^{2}$, the original dynamic model assumes that $C_{S}^{2} \operatorname{Pr}_{s g s}^{-1}$ is scale invariant and, therefore, $\beta_{\theta}=1$.

Minimising the error given by Eq. 14 using the least squares method, we obtain the optimal value of the lumped eddy-diffusivity coefficient as

$$
C_{S}^{2} \operatorname{Pr}_{\text {sgs }}^{-1}=\frac{\left\langle K_{i} X_{i}\right\rangle}{\left\langle X_{i} X_{i}\right\rangle}
$$

where, in the case of the Lagrangian dynamic model, the brackets \langle\rangle denote averaging along fluid pathlines.

The Lagrangian dynamic models given by Eqs. 11 and 17 provide a systematic way to optimise the values of the coefficients $C_{S}^{2}$ and $C_{S}^{2} \operatorname{Pr}_{s g s}^{-1}$ by calculating them directly from the resolved scales in the LES model. However, the standard (scale-invariant) dynamic models have been found to yield SGS dissipation rates (transfer rates of energy and scalar variance from resolved to subgrid scales) that are too small. As a result, these models produce unrealistic turbulence statistics (e.g., mean velocity and scalar profiles as well as turbulence spectra) in simulations of the neutral boundary-layer flow over homogeneous (Porté-Agel et al. 2000) 
and heterogeneous (Bou-Zeid et al. 2005; Stoll and Porté-Agel 2006) flat surfaces as well as the boundary layer over topography (Iizuka and Kondo 2004; Wan et al. 2007).

\subsection{The Scale-Dependent Lagrangian Dynamic Model}

Porté-Agel et al. (2000) developed a scale-dependent dynamic model, in which the assumption of scale invariance of the model coefficient is relaxed and the model coefficient is allowed to change with scale. Porté-Agel et al. (2000) assumed that $C_{S}^{2}$ can be expressed as a power-law function of $\Delta$, which implies that the scale-dependence parameter $\beta$ is no longer a constant value of 1 and needs to be determined dynamically. The exponent in the power law is directly related to the value of $\beta$.

To dynamically calculate the scale-dependence parameter $\beta$, a second test filter $\widehat{\Delta}$ needs to be employed. We typically take $\widehat{\Delta}=4 \Delta$, and use a caret to denote variables filtered at scale $4 \Delta$. The following equality is automatically satisfied once the power-law dependence of model coefficients with scale proposed by Porté-Agel et al. (2000) is assumed:

$$
\beta=C_{S}^{2}(4 \Delta) / C_{S}^{2}(2 \Delta)=C_{S}^{2}(2 \Delta) / C_{S}^{2}(\Delta) .
$$

The error incurred by using the eddy-viscosity model in the Germano identity between scales $\Delta$ and $\widehat{\Delta}$ becomes

$$
e_{i j}^{\prime}=L_{i j}^{\prime}-\frac{1}{3} \delta_{i j} L_{k k}^{\prime}-C_{S}^{2}(\Delta) M_{i j}^{\prime}
$$

where

$$
\begin{gathered}
L_{i j}^{\prime}=\widehat{\widetilde{u}_{i} \widetilde{u}_{j}}-\widehat{\widetilde{u}}_{i} \widehat{\widetilde{u}}_{j}, \\
M_{i j}^{\prime}=2 \Delta^{2}\left(\left|\widetilde{\widetilde{S} \mid \widetilde{S}_{i j}}-4^{2} \beta^{2}\right| \widehat{\widehat{S}} \mid \widehat{\widetilde{S}}_{i j}\right) .
\end{gathered}
$$

Minimising the error in Eq. 19 using the earlier mentioned minimisation method, we obtain the following equation for $C_{S}^{2}$ :

$$
C_{S}^{2}=\frac{\left\langle L_{i j}^{\prime} M_{i j}^{\prime}\right\rangle}{\left\langle M_{i j}^{\prime} M_{i j}^{\prime}\right\rangle} .
$$

Again, the brackets in Eq. 22 denote Lagrangian averaging over fluid pathlines. The scaledependence parameter $\beta$ can now be dynamically determined by combining Eqs. 11 and 22 .

For scalar fluxes, a similar scale-dependent dynamic procedure can be derived (PortéAgel 2004; Stoll and Porté-Agel 2006). In this case, the error associated with the use of the eddy-diffusivity model in the Germano identity between $\Delta$ and $\widehat{\Delta}$ becomes

$$
e_{i}^{\prime}=K_{i}^{\prime}-C_{S}^{2} \operatorname{Pr}_{s g s}^{-1}(\Delta) X_{i}^{\prime}
$$

where

$$
\begin{aligned}
& K_{i}^{\prime}=\widehat{\widetilde{\widetilde{u}}_{i} \widetilde{\theta}}-\widehat{\widetilde{u}}_{i} \widehat{\tilde{\theta}} \\
& X_{i}^{\prime}=\Delta^{2}\left(\left|\widehat{\widetilde{S} \mid \frac{\partial \tilde{\theta}}{\partial x_{i}}}-4^{2} \beta_{\theta}^{2}\right| \widehat{\widetilde{S}} \mid \frac{\partial \hat{\tilde{\theta}}}{\partial x_{i}}\right) .
\end{aligned}
$$


Again, the lumped model coefficient is assumed to have a power-law dependence with scale in the range of scales between the grid/filter scale and the second test filter scale so that

$$
\beta_{\theta}=C_{S}^{2} \operatorname{Pr}_{\text {sgs }}^{-1}(2 \Delta) / C_{S}^{2} \operatorname{Pr}_{\text {sgs }}^{-1}(\Delta)=C_{S}^{2} \operatorname{Pr}_{\text {sgs }}^{-1}(4 \Delta) / C_{S}^{2} \operatorname{Pr}_{\text {sgs }}^{-1}(2 \Delta) .
$$

The same method used with the first test filter is employed here to minimise the error in Eq. 23 locally backward along fluid pathlines, resulting in the following equation for $C_{S}^{2} \mathrm{Pr}_{s g s}^{-1}$ :

$$
C_{S}^{2} \operatorname{Pr}_{s g s}^{-1}=\frac{\left\langle K_{i}^{\prime} X_{i}^{\prime}\right\rangle}{\left\langle X_{i}^{\prime} X_{i}^{\prime}\right\rangle} .
$$

Combining Eqs. 27 and 17 results in a single equation from which the unknown scale-dependence parameter $\beta_{\theta}$ may be obtained dynamically. For more details on the scale-dependent Lagrangian dynamic procedure, see Stoll and Porté-Agel (2006).

\section{Numerical Experiments}

The experiment by Ross et al. (2004) was carried out in the wind tunnel at the Environmental Flow Research Laboratory (EnFlo), University of Surrey, U.K. The wind tunnel has a working section of $20 \mathrm{~m}$ length, $3.5 \mathrm{~m}$ width and $1.5 \mathrm{~m}$ height. The fan can produce flow speeds in the range between 0.3 and $4.5 \mathrm{~m} \mathrm{~s}^{-1}$. A range of multi-level heaters was used to generate thermal stratification, and by doing this, a maximum temperature gradient of approximately $80 \mathrm{~K} \mathrm{~m}^{-1}$ could be attained. To maintain a stable boundary layer, the floor was cooled by pumping cold water through pipes beneath the floor. Roughness elements $(20 \mathrm{~mm}$ high and $80 \mathrm{~mm}$ wide, separated laterally by $160 \mathrm{~mm}$ and downwind by $100 \mathrm{~mm}$, in a triple stagger) were placed over the entire floor, including the hill surface. The flow velocity was measured by a two-component fibre optic, He-Ne laser Doppler anemometer with burst spectrum analysers. The free-stream velocity was measured using an ultrasonic anemometer. Measurements of temperature fluctuation were carried out using cold wires and thermocouples.

In this numerical study, the large-eddy simulation code is a modified version of that described by Albertson and Parlange (1999), Porté-Agel et al. (2000) and Stoll and PortéAgel (2006). We use pseudospectral methods to compute spatial derivatives in the horizontal directions and second-order central finite differences in the vertical direction. For the entire computational domain, periodic boundary conditions are applied in the horizontal directions. Time advancement is carried out by using a second-order Adams Bashforth scheme.

A fixed stress-free lid is applied as the upper boundary condition at the top of the domain. The lower boundary condition consists of using similarity theory (Monin-Obukhov similarity) to calculate the instantaneous (filtered) surface shear stress and heat flux as a function of the resolved velocity and temperature at the lowest computational level. The surface temperature is maintained at $300.5 \mathrm{~K}$ to match the wind-tunnel conditions. In order to approximate the upstream velocity and temperature inflow boundary conditions in the wind-tunnel experiment (Ross et al. 2004), we employed a relaxation zone located upwind of the hill (see Fig. 1). The relaxation zone is set to smoothly bring the velocity and temperature fields to values corresponding to the upwind flat wind-tunnel boundary layer. These velocity and temperature fields used in the relaxation zone are obtained from separate simulations over a flat surface in the absence of the hill. In these simulations, we saved the cross-sectional velocity and temperature fields at the position corresponding to the end of the relaxation zone every 10 timesteps and used them as upstream inflow boundary conditions for simulations over the two-dimensional hill. The saved velocity and temperature fields are in good 


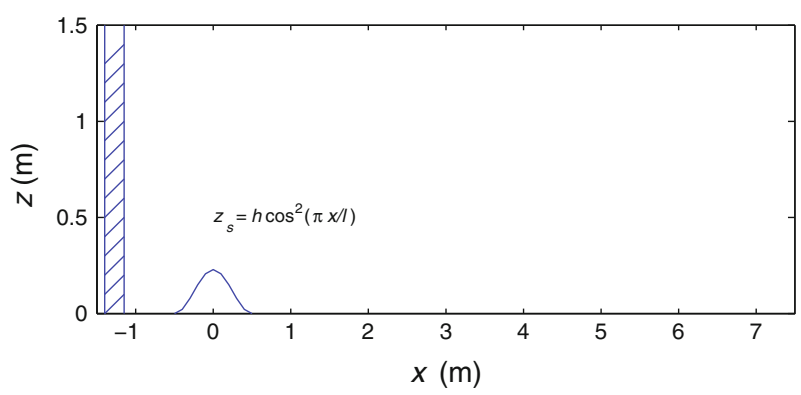

Fig. 1 Sketch of simulation domain and relaxation zone (shaded area)

agreement with the profiles measured by Ross et al. (2004) without the hill. In simulations over the two-dimensional hill, we used the relaxation zone to smoothly bring the velocity and temperature fields to the saved target fields every 10 timesteps. For simulations over a flat surface, a horizontal pressure gradient is exerted on the flow in the streamwise direction. The magnitude of this pressure gradient is set to match the free-stream wind speed of $U=$ $1.0 \mathrm{~m} \mathrm{~s}^{-1}$ measured in the wind-tunnel experiments. The use of a similar relaxation zone to impose an inflow boundary condition while maintaining the accuracy of pseudospectral LES codes has been successful in previous studies of turbulent transport in urban canopies (e.g., Tseng et al. 2006).

The simulated physical domain consists of the space around the two-dimensional hill with non-dimensional elevation

$$
z_{s} / L_{z}= \begin{cases}\left(h / L_{z}\right) \cos ^{2}(\pi x / l), & -l / 2 \leq x \leq l / 2 \\ 0, & \text { elsewhere }\end{cases}
$$

where $h / L_{z}$ is the normalised hilltop height, $z_{s} / L_{z}$ is the normalised domain surface height, $x$ and $l$ are the streamwise position and width of the hill $(l=1 \mathrm{~m})$ respectively. The maximum slope of the hill is $\pi h / l=0.72$, which shows the hill is steep. Figure 1 shows the simulation domain as well as the relaxation zone (shaded area).

The coordinate transformation developed by Clark (1977) has been used to transform the physical domain into a computational domain with flat surface. It is a terrain-following transformation of the form:

$$
\bar{z}=H\left(z-z_{s}\right) /\left(H-z_{s}\right),
$$

where $\bar{z}$ is the vertical position in the transformed system, $z$ is the actual elevation in the original system and $H$ denotes the top of the domain. After the transformation, the governing equations for momentum and temperature become:

$$
\begin{aligned}
\frac{\partial(\sqrt{G} \tilde{u})}{\partial t}+\sqrt{G} \widetilde{u} \frac{\partial \widetilde{u}}{\partial \bar{x}}+\sqrt{G} \widetilde{v} \frac{\partial \widetilde{u}}{\partial \bar{y}}+\sqrt{G} \widetilde{w_{c}} \frac{\partial \widetilde{u}}{\partial \bar{z}}+\frac{\partial\left(J_{3} \widetilde{P}\right)}{\partial \bar{x}}+\frac{\partial\left(J_{1} \widetilde{P}\right)}{\partial \bar{z}} \\
+\frac{\partial\left(J_{3} \tau_{11}\right)}{\partial \bar{x}}+\frac{\partial\left(J_{3} \tau_{12}\right)}{\partial \bar{y}}+\frac{\partial\left(\tau_{13}+J_{1} \tau_{11}+J_{2} \tau_{12}\right)}{\partial \bar{z}}=0, \\
\frac{\partial(\sqrt{G} \widetilde{v})}{\partial t}+\sqrt{G} \tilde{u} \frac{\partial \widetilde{v}}{\partial \bar{x}}+\sqrt{G} \widetilde{v} \frac{\partial \widetilde{v}}{\partial \bar{y}}+\sqrt{G} \widetilde{w_{c}} \frac{\partial \widetilde{v}}{\partial \bar{z}}+\frac{\partial\left(J_{3} \widetilde{P}\right)}{\partial \bar{y}}+\frac{\partial\left(J_{2} \widetilde{P}\right)}{\partial \bar{z}} \\
+\frac{\partial\left(J_{3} \tau_{12}\right)}{\partial \bar{x}}+\frac{\partial\left(J_{3} \tau_{22}\right)}{\partial \bar{y}}+\frac{\partial\left(\tau_{23}+J_{1} \tau_{12}+J_{2} \tau_{22}\right)}{\partial \bar{z}}=0,
\end{aligned}
$$




$$
\begin{aligned}
& \frac{\partial(\sqrt{G} \widetilde{w})}{\partial t}+\sqrt{G} \widetilde{u} \frac{\partial \widetilde{w}}{\partial \bar{x}}+\sqrt{G} \widetilde{v} \frac{\partial \widetilde{w}}{\partial \bar{y}}+\sqrt{G} \widetilde{w_{c}} \frac{\partial \widetilde{w}}{\partial \bar{z}}+\frac{\partial \widetilde{P}}{\partial \bar{z}}+\frac{\partial\left(J_{3} \tau_{13}\right)}{\partial \bar{x}} \\
& +\frac{\partial\left(J_{3} \tau_{23}\right)}{\partial \bar{y}}+\frac{\partial\left(\tau_{33}+J_{1} \tau_{13}+J_{2} \tau_{23}\right)}{\partial \bar{z}}-\sqrt{G} g \frac{\tilde{\theta}^{\prime}}{\theta_{0}}=0, \\
& \frac{\partial(\sqrt{G} \widetilde{\theta})}{\partial t}+\sqrt{G} \widetilde{u} \frac{\partial \widetilde{\theta}}{\partial \bar{x}}+\sqrt{G} \widetilde{v} \frac{\partial \widetilde{\theta}}{\partial \bar{y}}+\sqrt{G} \widetilde{w_{c}} \frac{\partial \widetilde{\theta}}{\partial \bar{z}}+\frac{\partial\left(J_{3} q_{1}\right)}{\partial \bar{x}}+\frac{\partial\left(J_{3} q_{2}\right)}{\partial \bar{y}} \\
& +\frac{\partial\left(q_{3}+J_{1} q_{1}+J_{2} q_{2}\right)}{\partial \bar{z}}=0,
\end{aligned}
$$

where $\bar{x}, \bar{y}, \bar{z}$ are the curvilinear coordinates in the computational domain, $\widetilde{u}, \widetilde{v}, \widetilde{w}$ are filtered velocities in the physical domain, $\widetilde{w_{c}}$ denotes the filtered vertical velocity in the computational domain, $\widetilde{\theta}$ is the filtered temperature, $\widetilde{P}$ represents the filtered pressure, $\sqrt{G}$ is the determinant of the Jacobian matrix of transformation, $J_{1}, J_{2}, J_{3}$ are non-zero components of the Jacobian matrix of transformation, $\tau_{11}, \tau_{12}, \tau_{13}, \tau_{22}, \tau_{23}, \tau_{33}$ are the SGS stresses, $q_{1}, q_{2}, q_{3}$ denote SGS heat fluxes, $g$ is the gravitational acceleration, $\widetilde{\theta_{0}}$ is a reference temperature, $\widetilde{\theta}^{\prime}$ is the fluctuation of the filtered temperature. Taking the divergence of the discretised momentum equations and applying the continuity equation, we obtain the pressure Poisson equation. The pressure Poisson equation is solved iteratively at every timestep until the solution converges. The computational domain is of size $9 \mathrm{~m} \times 1.5 \mathrm{~m} \times 1.5 \mathrm{~m}$, that is $(2 \pi, \pi / 3, \pi / 3)$ in non-dimensionalised form by using a length scale of $L_{z}=1.432 \mathrm{~m}$. The normalised effective surface roughness is $z_{0} / L_{z}=1.606 \times 10^{-3}$. The computational domain is divided into $180 \times 30 \times 120$ uniformly spaced grid points. The grid is staggered in the vertical direction, with the vertical velocity stored halfway between the other variables. The relaxation zone covers six grid points in the streamwise direction. A sponge layer is applied at the domain top to absorb possible gravity waves. The timestep used in the simulations is $0.002864 \mathrm{~s}$, and the duration of the simulation is $429 \mathrm{~s}$.

The temperature field of this stably-stratified boundary-layer flow is composed of two layers with different levels of stratification: a region of relatively weak stratification of about $10 \mathrm{~K} \mathrm{~m}^{-1}$ in the lowest $0.5 \mathrm{~m}$, and a region of stronger stratification (about $40 \mathrm{~K} \mathrm{~m}^{-1}$ ) above $(z>0.5 \mathrm{~m})$. The simulations are run for a period of time long enough to guarantee quasisteady flow conditions and statistical convergence of the results, presented in the next section.

\section{Results}

Figure $2 \mathrm{a}$ shows a contour plot of the streamwise velocity $u$ (in $\mathrm{m} \mathrm{s}^{-1}$ ) measured during the wind-tunnel experiment in a vertical plane perpendicular to the two-dimensional hill (Ross et al. 2004). Figure 2b-d show similar contour plots of averaged (in time and spanwise direction) streamwise velocities simulated using large-eddy simulation (LES) with different subgrid-scale (SGS) models: the Smagorinsky model (Fig. 2b), the Lagrangian dynamic model (Fig. 2c), and the scale-dependent Lagrangian dynamic model (Fig. 2d). Comparison of Fig. 2a and $\mathrm{b}$ reveals important differences between the measured velocity field and the simulated velocity obtained with the Smagorinsky model. In particular, the Smagorinsky model grossly overpredicts the size of the recirculation region on the lee side of the hill. The location of the separation point predicted by the Smagorinsky model is around the hill top. Moreover, the model underpredicts the velocity magnitude right above the hill top. 

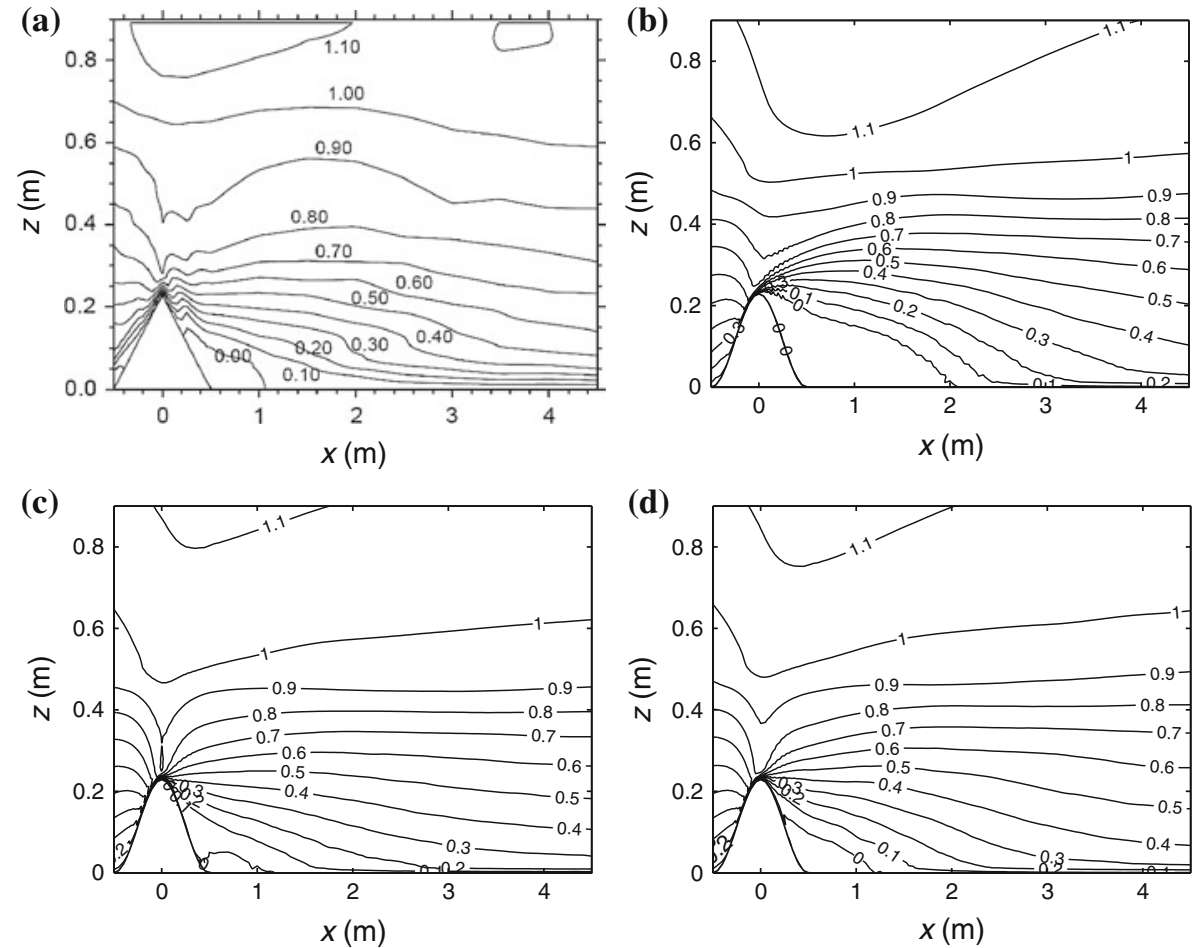

Fig. 2 Contour plots of streamwise velocity $u$ (in $\mathrm{m} \mathrm{s}^{-1}$ ) in a vertical plane perpendicular to the hill: a Wind-tunnel measurements (Ross et al. 2004), b Smagorinsky model, c Lagrangian dynamic model, d scale-dependent Lagrangian dynamic model

Figure 2c and $\mathrm{d}$ show that both dynamic models improve the velocity prediction with respect to the Smagorinsky model. The Lagrangian dynamic model, however, is found to underestimate the size of the recirculation region. From Fig. 2c, we see that the separation point is near the downwind foot of the hill. Moreover, this model overestimates the magnitude of the streamwise velocity right above the hill crest. A similar velocity overestimation from the Lagrangian dynamic model was reported in numerical simulations of neutral turbulent flow over two-dimensional topography (Iizuka and Kondo 2004; Wan et al. 2007). Overall, the scale-dependent Lagrangian dynamic model provides a more accurate prediction of the streamwise velocity field (Fig. 2d), compared with the Smagorinsky model and the scale-invariant dynamic model. In particular, the recirculation region predicted by the scale-dependent dynamic model matches more closely that measured in the wind-tunnel experiment (Fig. 2a). The separation point is about halfway downwind from the hill crest.

Figure 3a shows the contour plot of momentum flux $\overline{u^{\prime} w^{\prime}}\left(\mathrm{m}^{2} \mathrm{~s}^{-2}\right)$ measured during the wind-tunnel experiment in a vertical plane perpendicular to the two-dimensional hill (Ross et al. 2004). Figure $3 \mathrm{~b}-\mathrm{d}$ show similar contour plots of time-averaged momentum flux $\overline{u^{\prime} w^{\prime}}$ (resolved stress plus subgrid-scale stress) simulated using LES with different SGS models: the Smagorinsky model (Fig. 3b), the Lagrangian dynamic model (Fig. 3c), and the scaledependent Lagrangian dynamic model (Fig. 3d). From these figures, we see that, because of the very strong stratification (about $40 \mathrm{~K} \mathrm{~m}^{-1}$ ) in the upper part of the domain (above $0.5 \mathrm{~m}$ ), turbulence was largely confined to the lower part of the domain. By comparing the 

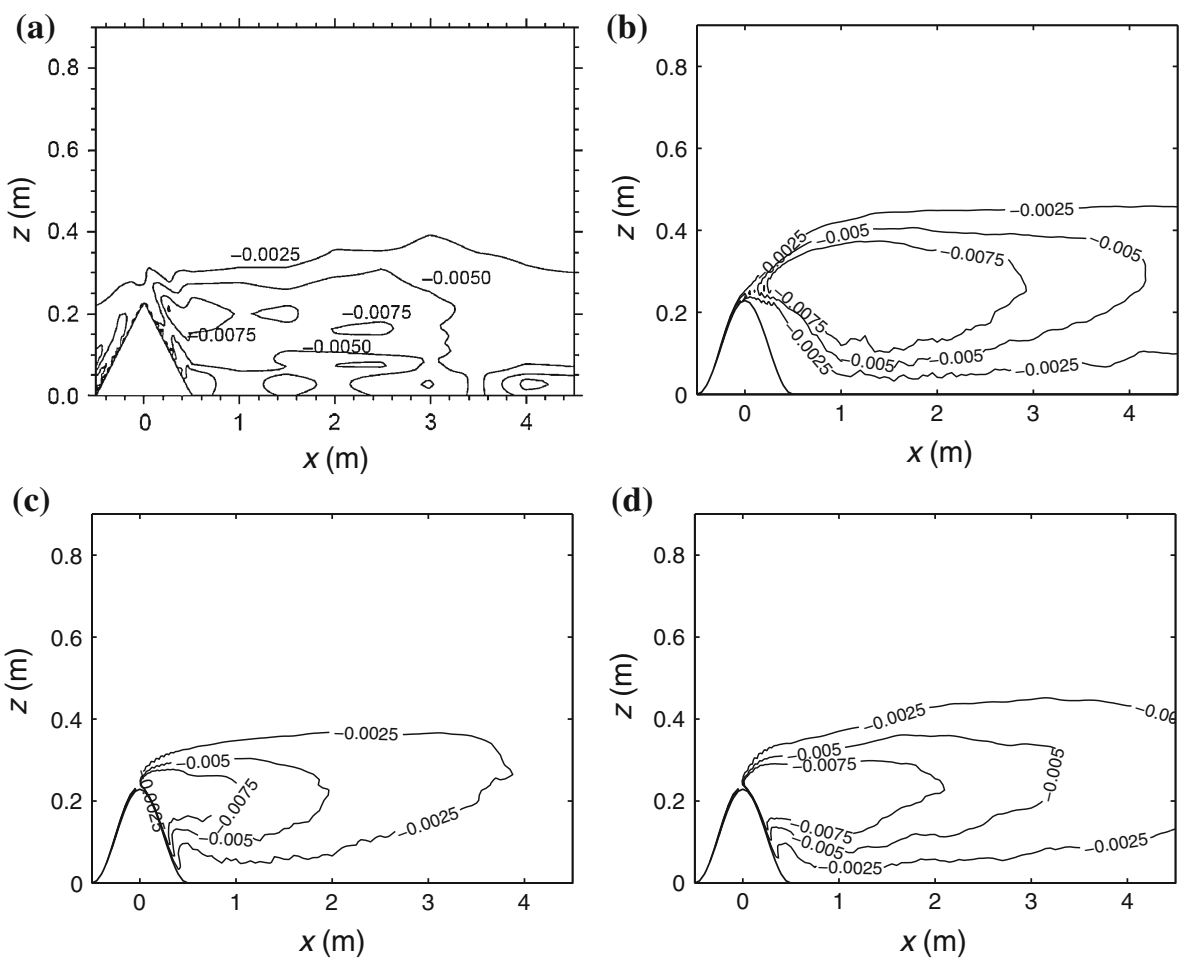

Fig. 3 Contour plots of momentum flux $\overline{u^{\prime} w^{\prime}}\left(\right.$ in $\mathrm{m}^{2} \mathrm{~s}^{-2}$ ) in a vertical plane perpendicular to the hill: a Wind-tunnel measurements (Ross et al. 2004), b Smagorinsky model, c Lagrangian dynamic model, d scale-dependent Lagrangian dynamic model

contour line of value $-0.005 \mathrm{~m}^{2} \mathrm{~s}^{-2}$ in Fig. 3a and b, the Smagorinsky model (Fig. 3b) is found to somewhat overestimate the size of the wake region downwind of the hill, which leads to a larger prediction of $\overline{u^{\prime} w^{\prime}}$ values in the domain. The Lagrangian dynamic model clearly underpredicts the size of the wake region behind the hill. Note that in Fig. $3 \mathrm{c}$ the region with flux values above $0.005 \mathrm{~m}^{2} \mathrm{~s}^{-2}$ in magnitude is much smaller than the region obtained from wind-tunnel measurements (Fig. 3a) and from the scale-dependent Lagrangian dynamic model (Fig. 3d). The scale-dependent Lagrangian dynamic model gives a more accurate prediction of the wake region behind the hill.

The dynamically calculated values of the eddy-viscosity model coefficient $C_{S}^{2}$ obtained using the Lagrangian dynamic and scale-dependent Lagrangian dynamic models are presented in Fig. 4a and b, respectively. The values represent averages in the spanwise direction and in time. In the upper portion of the domain, both models yield relatively low values of $C_{S}^{2}$ due to the very strong flow stratification in that region. This is consistent with previous studies that show $C_{S}^{2}$ decreasing with increasing stability (Porté-Agel et al. 2001a; Kleissl et al. 2003, 2006). The coefficients obtained with both dynamic models decrease near the surface to account for the reduction in the characteristic scales of the turbulence in that region of the flow. In addition, strong variability of the dynamically calculated $C_{S}^{2}$ is found around the crest of the hill, where the flow is heterogeneous in both the streamwise and vertical directions. Smaller values of $C_{S}^{2}$ are obtained over the upwind side of the hill crest, where the flow undergoes strong straining; larger $C_{S}^{2}$ values are found in the downhill side of the 

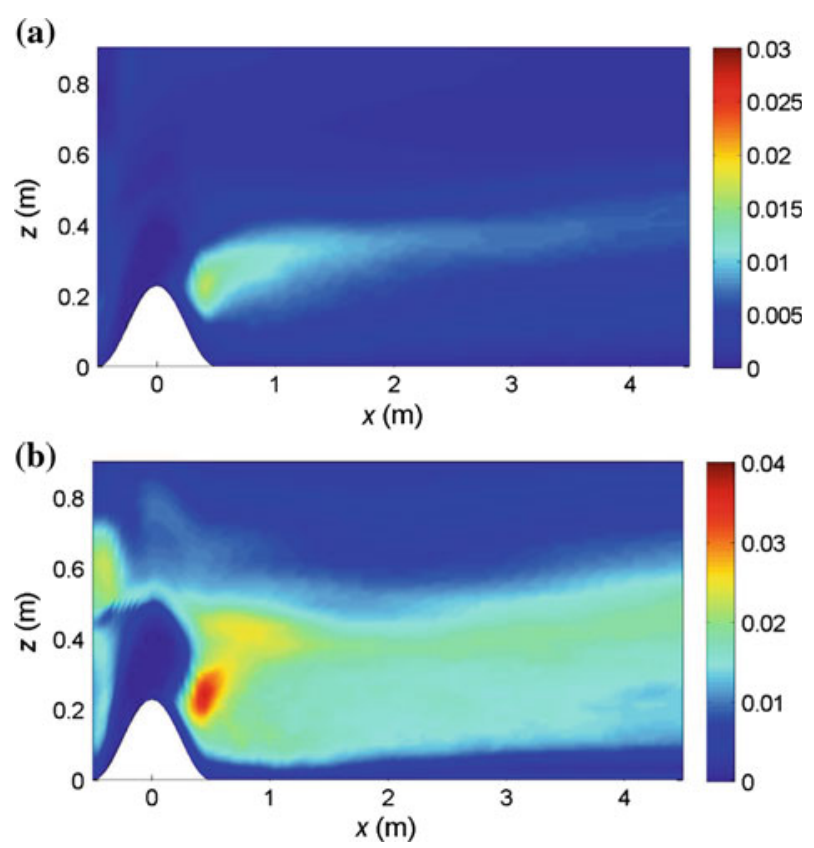

Fig. 4 Smagorinsky coefficient $C_{S}^{2}$ calculated dynamically with: a the Lagrangian dynamic model, b the scale-dependent Lagrangian dynamic model

hill, where the flow detaches from the surface, recirculates and experiences much smaller straining.

The values of the eddy-diffusivity model coefficient $C_{S}^{2} \operatorname{Pr}_{s g s}^{-1}$ obtained using the Lagrangian dynamic and scale-dependent Lagrangian dynamic model are presented in Fig. 5a and $b$, respectively. The values represent averages in the spanwise direction and in time. Similar to $C_{S}^{2}$, the value of the lumped coefficient $C_{S}^{2} \operatorname{Pr}_{s g s}^{-1}$ is small in the upper part of the domain to account for the reduction in the characteristic scale of the turbulence associated with the strong stratification of the flow (Porté-Agel et al. 2001a; Kleissl et al. 2003, 2006). In the lower part, $C_{S}^{2} \mathrm{Pr}_{s g s}^{-1}$ is found to decrease as the surface is approached in order to account for the reduction in the characteristic scale of the turbulence near the surface. Horizontal variations of the lumped coefficient $C_{S}^{2} \operatorname{Pr}_{s g s}^{-1}$ are also manifest in the figures.

Figure $6 \mathrm{a}$ and $\mathrm{b}$ show the scale-dependence parameters $\beta\left(=C_{S}^{2}(2 \Delta) / C_{S}^{2}(\Delta)\right)$ and $\beta_{\theta}\left(=C_{S}^{2} \operatorname{Pr}_{s g s}^{-1}(2 \Delta) / C_{S}^{2} \operatorname{Pr}_{s g s}^{-1}(\Delta)\right)$ obtained with the scale-dependent Lagrangian dynamic model. As expected, the scale-dependence parameters become smaller as the surface is approached due to increased shear and anisotropy of the flow. The smallest values of $\beta$ and $\beta_{\theta}$ are found near the upwind side of the hill crest, where the mean shear and anisotropy of the flow are stronger; downwind of the hill, where the flow separates and becomes less anisotropic, the scale-dependence coefficients are larger. These overall trends are consistent with the results obtained for $\beta$ in simulations of a neutral boundary layer over a sinusoidal surface (Wan et al. 2007).

Figure $7 \mathrm{a}-\mathrm{c}$ show contour plots of the averaged (in time and spanwise direction) resolved turbulent kinetic energy (in $\mathrm{m}^{2} \mathrm{~s}^{-2}$ ) obtained with the three SGS models under consideration. On the upwind side of the hill crest, the Lagrangian dynamic model predicts 

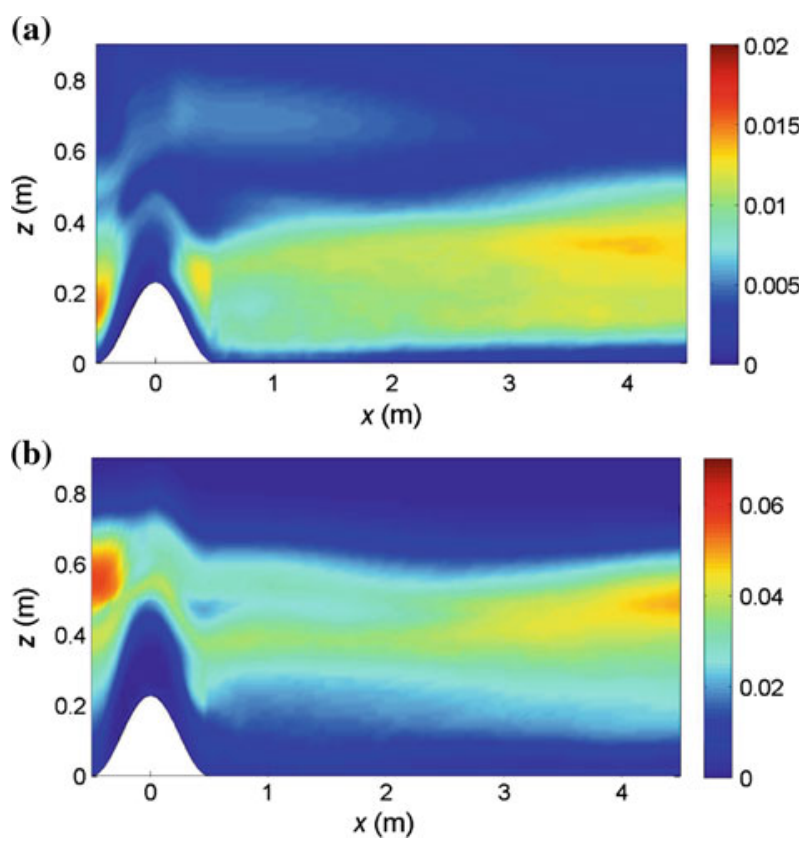

Fig. 5 Lumped eddy-diffusivity coefficient $C_{S}^{2} \operatorname{Pr}_{s g s}^{-1}$ calculated dynamically with: a the Lagrangian dynamic model, $\mathbf{b}$ the scale-dependent Lagrangian dynamic model
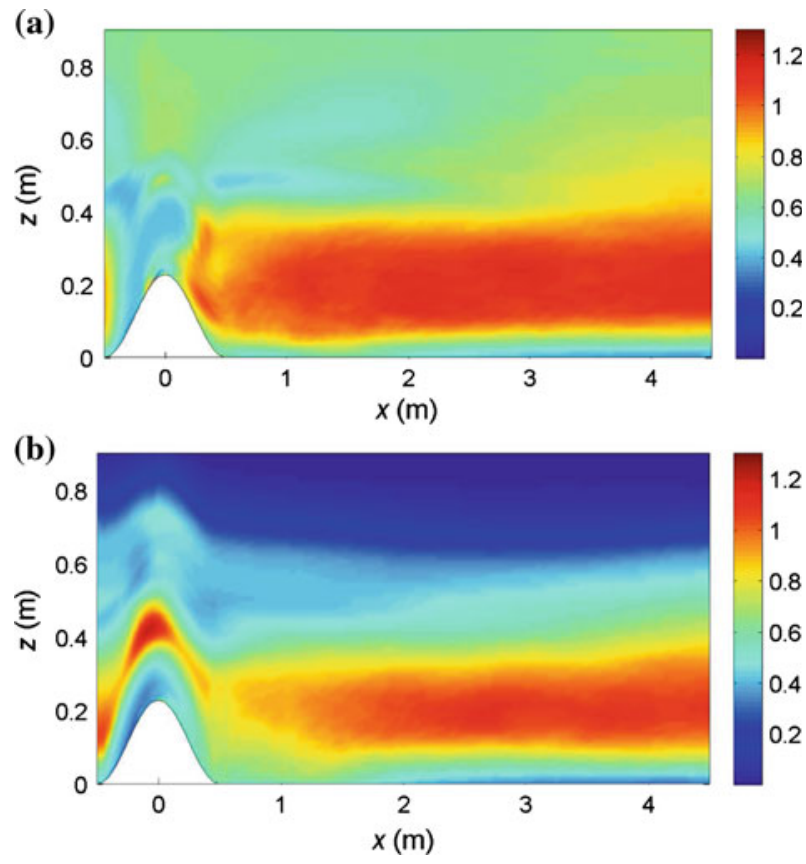

Fig. 6 Scale-dependence parameters $\beta$ (a) and $\beta_{\theta}$ (b) obtained with the scale-dependent Lagrangian dynamic model 

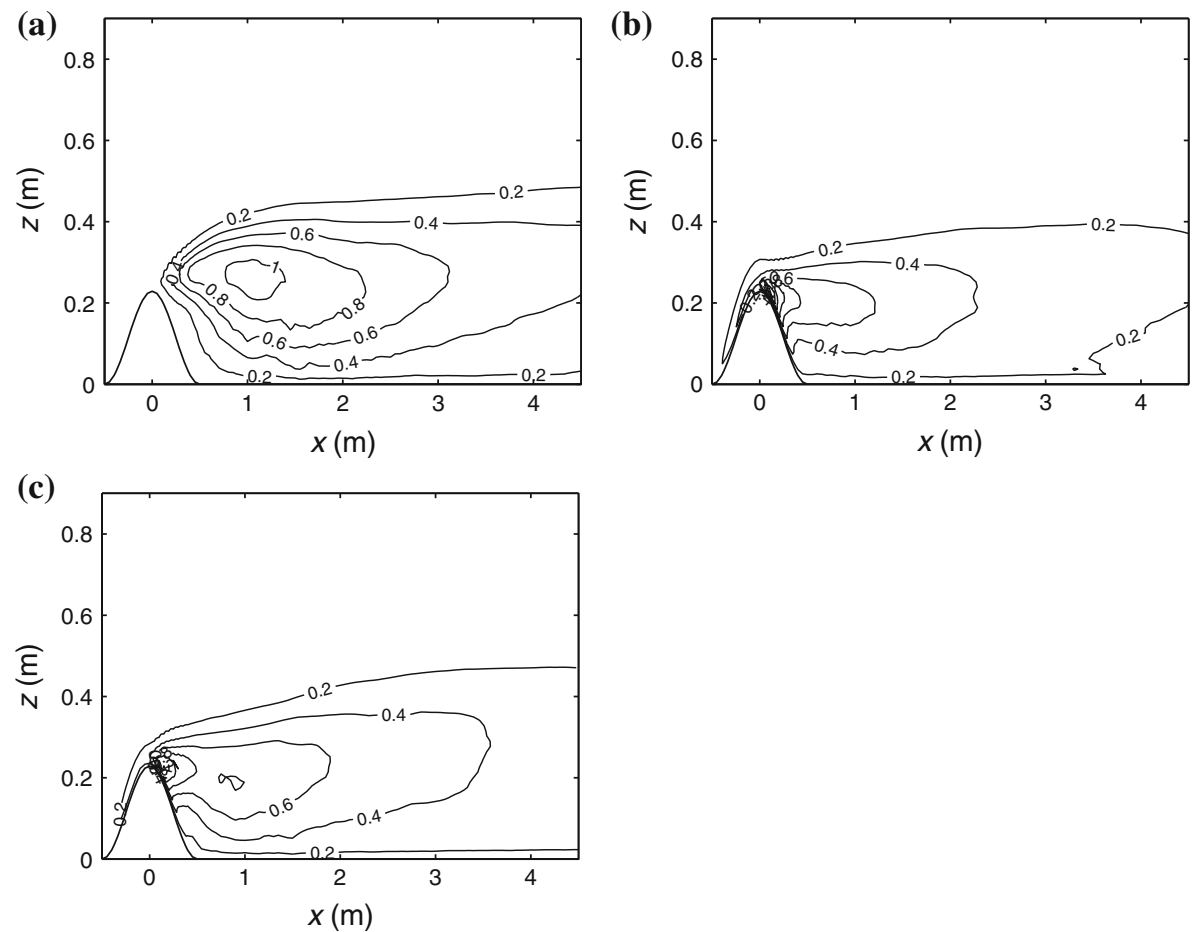

Fig. 7 Contour plots of resolved turbulent kinetic energy (in $\mathrm{m}^{2} \mathrm{~s}^{-2}$ ) in a vertical plane perpendicular to the hill: a Smagorinsky model, b Lagrangian dynamic model, c scale-dependent Lagrangian dynamic model

the largest kinetic energy among the three models, while the Smagorinsky model yields the smallest. This is in agreement with previous studies (e.g., Porté-Agel et al. 2000) that show that the Lagrangian dynamic model is insufficiently dissipative in the near-ground region, yielding too much resolved kinetic energy. In contrast, the Smagorinsky model is known to be too dissipative near the ground. The scale-dependent dynamic model has been found to provide more realistic SGS dissipation rates in simulations of the homogeneous boundary layer over flat terrain (e.g., Porté-Agel et al. 2000). In the downwind wake region, away from the hill, the magnitude of the turbulent kinetic energy obtained with the Lagrangian dynamic model is clearly smaller than that obtained with the Smagorinsky model and the scale-dependent Lagrangian dynamic model. The Smagorinsky model yields the greatest turbulent kinetic energy among the three models. This is in accordance with previous studies (e.g., Murakami et al. 1996; Iizuka and Kondo 2004), which reported that the standard Smagorinsky model overestimates the turbulent kinetic energy in the free shear layer behind the obstacle. The differences in the prediction of the resolved turbulent kinetic energy in the wake region, and the size of that region can be explained in part by the differences in the simulated flow on the upwind side of the hill crest. In order to better understand that effect, contour plots of the averaged (in time and spanwise direction) vertical velocity component are shown in Fig. 8 for the three models under consideration. It is clear that the Smagorinsky model leads to a relatively large prediction of the vertical velocity over the upwind side of the hill crest (Fig. 8a). This is consistent with the underestimation of the streamwise velocity in that region (Fig. 2b), and leads to an excessive upward deflection of the flow. This, in turn, leads to large resolved kinetic energy in the downstream region of the hill (Fig. 7a) 

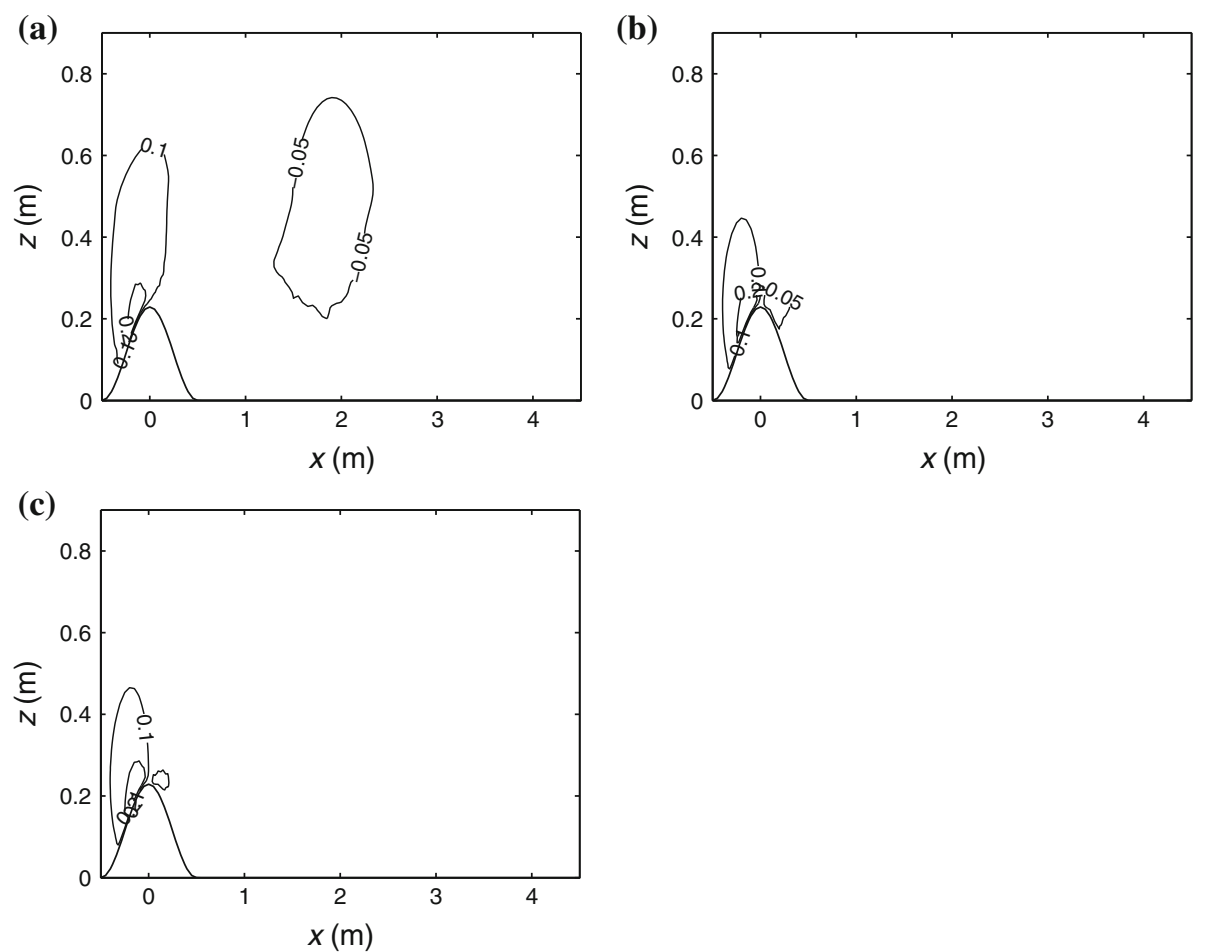

Fig. 8 Contour plots of vertical velocity $w$ (in $\mathrm{m} \mathrm{s}^{-1}$ ) in a vertical plane perpendicular to the hill: a Smagorinsky model, b Lagrangian dynamic model, c scale-dependent Lagrangian dynamic model

and the formation of unrealistically large wake and recirculation regions there. This effect is also reflected in the distribution of mean temperature predicted with the Smagorinsky model (Fig. 9a), which shows a stronger upward deflection compared with those obtained with the two dynamic models. In contrast, the Lagrangian dynamic model predicts a relatively larger horizontal velocity (Fig. 2c) and smaller vertical velocity (Fig. 8b) on the upwind side of the hill crest, which produces a smaller upward deflection of the flow. This translates into smaller vertical mixing, resolved kinetic energy (Fig. 7b) and wake size in the downwind region of the hill. This is also consistent with the contours of temperature, which show a smaller upward deflection around the hill (Fig. 9b), compared with the Smagorinsky model (Fig. 9a).

It is important to point out that our results near the upwind side of the hill crest agree with previous studies that found that the scale-dependent dynamic model has improved SGS dissipation characteristics in the surface layer, compared with the Smagorinsky and scale-invariant dynamic models. Near the surface, those models are too dissipative and not dissipative enough, respectively (e.g., Porté-Agel et al. 2000; Bou-Zeid et al. 2005; Wan et al. 2007; Stoll and Porté-Agel 2008). In general, the results presented here also agree with previous studies that found the scale-dependent dynamic model yields improved predictions of the flow statistics (e.g., average velocity and turbulent fluxes), not only in the surface layer, but throughout most of the computational domain. 

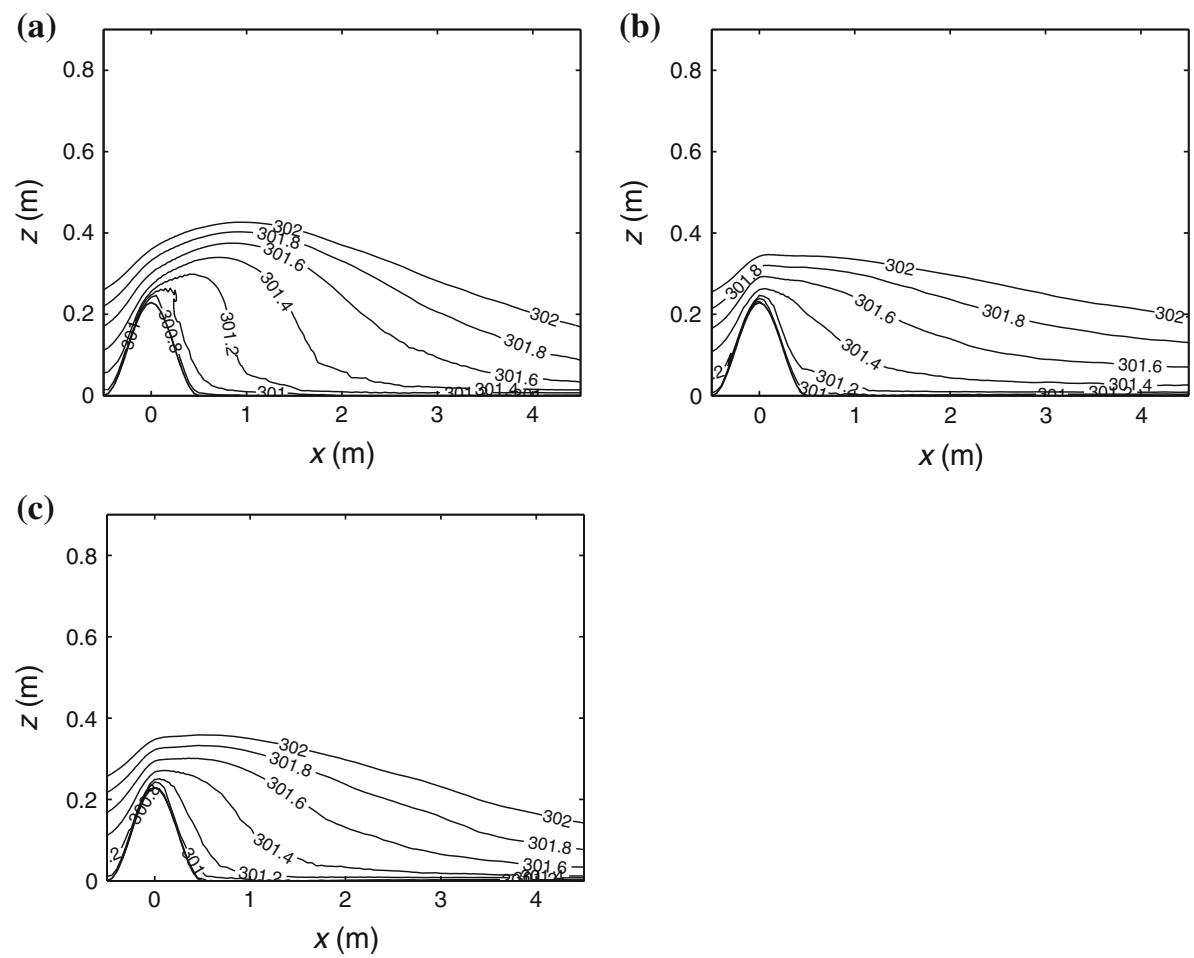

Fig. 9 Contour plots of temperature $\theta$ (in K) in a vertical plane perpendicular to the hill: a Smagorinsky model, b Lagrangian dynamic model, c scale-dependent Lagrangian dynamic model

\section{Summary}

Large-eddy simulation (LES) has been used to investigate stably-stratified turbulent boundary-layer flow over a steep two-dimensional hill. Three different types of subgrid-scale (SGS) models for both the SGS stresses and SGS heat fluxes are tested: (a) the Smagorinsky model, (b) the Lagrangian dynamic model, and (c) the scale-dependent Lagrangian dynamic model (Stoll and Porté-Agel 2006). Simulation results obtained with the different models are compared with turbulence statistics obtained from experiments conducted in the environmental wind tunnel at the Environmental Flow Research Laboratory (EnFlo), University of Surrey in the U.K. (Ross et al. 2004).

In this stably-stratified turbulent boundary-layer flow simulation, the standard (nondynamic) Smagorinsky model yields relatively poor predictions of the turbulent statistics, arguably due to its failure to adjust the model coefficients to capture the spatial variations of the length scales in the SGS eddy viscosity and eddy diffusivity. In particular, this model is found to be too dissipative near the surface on the upwind side of the hill crest, and to overpredict the upward deflection of the flow in that region. This is consistent with previous studies of neutral boundary-layer flow over topography (Wan et al. 2007), and is associated with an overprediction of the size of the simulated wake region.

Dynamic models offer a more systematic means of optimising the local value of the eddy viscosity and eddy diffusivity SGS model coefficients by computing them dynamically at every timestep and position in the flow based on the dynamics of the smallest resolved scales 
(between the grid/filter scale and a test filter scale). As a result, both the Lagrangian dynamic model and the scale-dependent Lagrangian dynamic model show improved predictions with respect to the standard non-dynamic models. However, the Lagrangian dynamic model is not dissipative enough and overestimates the horizontal velocity above the hill crest. This is consistent with the results from previous LES studies of neutral boundary-layer flow over two-dimensional hills (Iizuka and Kondo 2004; Wan et al. 2007). This model is found to underpredict the size of the downwind wake region.

By relaxing the assumption of scale invariance in the dynamic model, the scale-dependent dynamic model (Porté-Agel et al. 2000; Stoll and Porté-Agel 2006) is able to dynamically (without any parameter tuning) capture the scale dependence of the model coefficient associated with regions of the flow with strong shear and/or thermal stratification. Our results show that this procedure substantially improves the simulation results with respect to both the standard (non-dynamic) eddy-viscosity/diffusivity model and the scale-invariant dynamic model.

Acknowledgements This work was supported by NSF (grants EAR-0537865 and EAR-0120914 as part of the National Center for Earth-surface Dynamics) and NASA (grant NNG06GE256). Computer resources were provided by the Minnesota Supercomputing Institute.

\section{References}

Albertson JD, Parlange MB (1999) Surface length scales in shear stress: implications for land-atmosphere interactions over complex terrain. Water Resour Res 35:2121-2132

Bardina J, Ferziger JH, Reynolds WC (1983) Improved turbulence models based on large eddy simulation of homogeneous, incompressible, turbulent flows. PhD thesis; Rep. TF-19. Thermosciences Division, Department of Mechanical Engineering, Stanford University

Beare RJ et al (2006) An intercomparison of large-eddy simulations of the stable boundary layer. BoundaryLayer Meteorol 118:247-272. doi:10.1007/s10546-004-2820-6

Bou-Zeid E, Meneveau C, Parlange MB (2005) A scale-dependent Lagrangian dynamic model for large eddy simulation of complex turbulent flows. Phys Fluids 17:025105

Bou-Zeid E, Vercauteren N, Parlange MB, Meneveau C (2008) Scale dependence of subgrid-scale model coefficients: an a priori study. Phys Fluids 20:115106

Brown AR, Hobson JM, Wood N (2001) Large-eddy simulation of neutral turbulent flow over rough sinusoidal ridges. Boundary-Layer Meteorol 98:411-441

Calhoun RJ, Street RL (2001) Turbulent flow over a wavy surface: neutral case. J Geophys Res 106:9277-9293

Calhoun RJ, Street RL, Koseff JR (2001) Turbulent flow over a wavy surface: stratified case. J Geophys Res 106:9295-9310

Canuto VM, Cheng Y (1997) Determination of the Smagorinsky-Lilly constant $C_{s}$. Phys Fluids 9:1368-1378

Clark T (1977) A small-scale dynamic model using a terrain-following coordinate transformation. J Comput Phys 24:186-215

Deardorff JW (1970) A numerical study of three-dimensional turbulent channel flow at large Reynolds numbers. J Fluid Mech 41:453-480

Germano M, Piomelli U, Moin P, Cabot W (1991) A dynamic subgrid-scale eddy viscosity model. Phys Fluids A 3(7):1760-1765

Gong W, Taylor PA, Dörnbrack A (1996) Turbulent boundary-layer flow over fixed aerodynamically rough two-dimensional sinusoidal waves. J Fluid Mech 312:1-37

Henn DS, Sykes RI (1999) Large-eddy simulation of flow over wavy surfaces. J Fluid Mech 383:75-112

Horiuti K (1993) A proper velocity scale for modelling subgridscale eddy viscosities in large-eddy simulation. Phys Fluids A 5(1):146-157

Iizuka S, Kondo H (2004) Performance of various sub-grid scale models in large-eddy simulation of turbulent flow over complex terrain. Atmos Environ 38:7083-7091

Kang HS, Meneveau C (2002) Universality of large eddy simulation model parameters across a turbulent wake behind a heated cylinder. J Turbul. doi:10.1088/1468-5248/3/1/032

Kleissl J, Meneveau C, Parlange MB (2003) On the magnitude and variability of subgrid-scale eddy-diffusion coefficients in the atmospheric surface layer. J Atmos Sci 60:2372-2388 
Kleissl J, Kumar V, Meneveau C, Parlange MB (2006) Numerical study of dynamic Smagorinsky models in large-eddy simulation of the atmospheric boundary layer: validation in stable and unstable conditions. Water Resour Res. doi:10.1029/2005WR004685

Lilly DK (1967) The representation of small-scale turbulence in numerical simulation experiments. In: Proceedings of IBM scientific computing symposium on environmental sciences, White Plains, NY, IBM Data Processing Division, p 195

Lilly DK (1992) A proposed modification of the Germano subgrid-scale closure method. Phys Fluids A 4(3):633-635

Mason PJ, Derbyshire SH (1990) Large-eddy simulation of the stably-stratified atmospheric boundary layer. Boundary-Layer Meteorol 53:117-162

Mason PJ, Thomson DJ (1992) Stochastic backscatter in large-eddy simulations of boundary layers. J Fluid Mech 242:51-78

Meneveau C, Lund T, Cabot W (1996) A Lagrangian dynamic subgrid-scale model of turbulence. J Fluid Mech 319:353-385

Moin P, Kim J (1982) Numerical investigation of turbulent channel flow. J Fluid Mech 118:341-377

Moin P, Squires K, Cabot W, Lee S (1991) A dynamic subgrid-scale model for compressible turbulence and scalar transport. Phys Fluids A 3(11):2746-2757

Murakami S, Mochida A, Ooka R, Kato S, Iizuka S (1996) Numerical prediction of flow around a building with various turbulence models: comparison of k- $\epsilon$ EVM, ASM, DSM, and LES with wind tunnel tests. ASHRAE Trans 102:741-753

Piomelli U, Moin P, Ferziger JH (1988) Model consistency in large eddy simulation of turbulent channel flows. Phys Fluids 31:1884-1891

Porté-Agel F (2004) A scale-dependent dynamic model for scalar transport in large-eddy simulations of the atmospheric boundary layer. Boundary-Layer Meteorol 112:81-105

Porté-Agel F, Meneveau C, Parlange MB (2000) A scale-dependent dynamic model for large-eddy simulation: application to a neutral atmospheric boundary layer. J Fluid Mech 415:261-284

Porté-Agel F, Pahlow M, Meneveau C, Parlange MB (2001a) Atmospheric stability effect on subgrid-scale physics for large-eddy simulation. Adv Water Res 24:1085-1102

Porté-Agel F, Parlange MB, Meneveau C, Eichinger WE (2001b) A priori field study of the subgrid-scale heat fluxes and dissipation in the atmospheric surface layer. J Atmos Sci 58:2673-2698

Ross AN, Arnold S, Vosper SB, Mobbs SD, Dixon N, Robins AG (2004) A comparison of wind tunnel experiments and numerical simulations of neutral and stratified flow over a hill. Boundary-Layer Meteorol 113:427-459

Smagorinsky J (1963) General circulation experiments with the primitive equations: I. The basic experiment. Mon Weather Rev 91:99-164

Stoll R, Porté-Agel F (2006) Dynamic subgrid-scale models for momentum and scalar fluxes in large-eddy simulation of neutrally stratified atmospheric boundary layers over heterogeneous terrain. Water Resour Res. doi:10.1029/2005WR003989

Stoll R, Porté-Agel F (2008) Large-eddy simulation of the stable atmospheric boundary layer using dynamic models with different averaging schemes. Boundary-Layer Meteorol 126:1-28

Tseng YH, Meneveau C, Parlange MB (2006) Modeling flow around bluff bodies and predicting urban dispersion using large eddy simulation. Environ Sci Technol 40:2653-2662

Wan F, Porté-Agel F, Stoll R (2007) Evaluation of dynamic subgrid-scale models in large-eddy simulations of neutral turbulent flow over a two-dimensional sinusoidal hill. Atmos Environ 41:2719-2728 